\title{
Minimal Repair Redundancy for Coherent System in its Signatures Representation
}

\author{
Vanderlei da Costa Bueno \\ Institute of Mathematics and Statistics, São Paulo University,Cx. Postal 66.281 05389-970, São Paulo, Brazil \\ E-mail: bueno@ime.usp.br \\ Received February 24, 2011; revised March 20, 2011; accepted March 23, 2011
}

\begin{abstract}
In this paper we discuss how to maintain the signature representation of a coherent system through a minimal repair redundancy. In a martingale framework we use compensator transforms to identify how the components minimal repairs affect the order statistics in the signature representation.
\end{abstract}

Keywords: System signature; Dynamic system signature; Coherent systems; Point processes martingales.

\section{Introduction}

The signature of a coherent system, as in [1], with independent andidentically distributed component lifetimes, as deifned by Samaniego, [2], is a vector whose $i$-th coordinate is the probability that the $I$-th component failure is fatal for the system.

The key feature of system signatures that makes them broadly useful in reliabilityanalysis is the fact that, in the context of independent and identically distributed (i.i.d.) absolutely continuous components lifetimes, they are distribution free measures of system quality, depending solely on the design characteristics of the system and independent of the behavior of the systems components

A detailed treatment of the theory and applications of system signatures may be found in Samaniego, [2]. This reference gives detailed justification for the i.i.d. assumption used in the definition of system signatures. By the way there are a host of applications in which the i.i.d. assumption is appropriate, ranging from batteries in lighting, to wafers or chips in a digital computer to the subsystem of spark plugs in an automobile engine.

Formally the definition is:

Let $T$ be the lifetime of a coherent system of order $n$, with components lifetimes $T_{1}, \ldots, T_{n}$, which are independent and identically distributed random variables with absolutely continuous distribution $F$. Then the signature vectorais defined as

$$
\alpha=\left(\alpha_{1}, \cdots, \alpha_{n}\right)(1)
$$

where $\alpha_{\mathrm{i}}=P\left(T=T_{(i)}\right)$ and the $\left\{T_{(i)}, 1 \leq i \leq n\right\}$ are the or- der statistics of $\left\{T_{i}, 1 \leq i \leq n\right\}$.

Clearly, under such conditions, $\left\{T=T_{i}\right\}, 1 \leq i \leq n$ is an almost sure $(P$-a.s. $)$ partition of the probability space, with probability $P$, and

$$
\begin{aligned}
P(T \leq t) & =\sum_{i=1}^{n} P\left(T=T_{(i)}\right) P\left(T_{(i)} \leq t \mid T=T_{(i)}\right) \\
& =\sum \alpha_{i} P\left(T_{(i)} \leq t\right)
\end{aligned}
$$

Samaniego [3], Kochar, et al. [4] and Shaked and Suarez-Llorens [5], extended thesignature concept to the case where the components lifetimes $T_{1}, \ldots, T_{n}$, of a system are exchangeable (i.e. the joint distribution function, $F\left(t_{1}, \ldots, t_{n}\right)$, of $\left(T_{1}, \ldots, T_{n}\right)$ is the same for any permutation of $\left(t_{1}, \ldots, t_{n}\right)$, an interesting and practical situation in reliability theory.

Concerning an improvement to system reliability, in its signature representation, through a redundancy operation of its components, and in view of the identically (exchangeable) distribution component lifetimes conditions, to maintain a system with its structural relation $P(T \leq t)=\sum \alpha_{i} P\left(T_{(i)} \leq t\right)$, we choose to apply the minimal repair redundancy. Intuitively the minimal repair redundancy gives to component $i$ an additional lifetime as it had just before the failure. Clearly, in the case of independent component lifetimes the whole system is returned to the state it had just before the failure.

A minimal redundancy of a lifetime $T$ produces the sum $T+S$ where $S$ is called spare lifetime and

$$
P(S>t \mid T=s)=P(T>t+s \mid T>s)(3)
$$


If the distribution function of $T$ is $F(t)=1-\bar{F}(t)$, the resulting lifetime $T+S$ hasthe distribution function $P(T+S \leq t)=1-P(T+S>t)$, where

$$
\begin{aligned}
P(T+S>t) & =P(T>t)+\int_{0}^{t} P(T+S>t \mid T=s) \mathrm{d} F(s) \\
& =\bar{F}(t)-\bar{F}(t) \ln \bar{F}(t)
\end{aligned}
$$

However in the context of system signature the approach of minimal repairs is not so clear: What are the effects of the independent (exchangeable) component lifetimes minimal repair in the ordered statistics and in the signatures itself? To answer such a question we consider dynamics signatures, as in a recent work by Bueno [6], in a general set up, under a complete information level, where the dependence (exchangeability) can be considered and the redundancy operations can be set through a compensator transform.

\section{Dynamic System Signature}

We consider, as in [6], the system evolution on time under a complete information level. In this fashion, if the components lifetimes are absolutely continuous, independent and identically distributed, the expected dynamic system signature enjoy the special property that they are independent of both the distribution $F$ and the time $t$. This fact has significance beyond the mere simplicity and tractability of the signature vector, reflect only characteristics of the corresponding system design and may be used as proxies for system designs in the comparison of system performance. Also the dynamic system signature actualizes itself under the system evolution on time recovering the dynamical system signature in the set $\left\{T_{(i)} \leq t<T_{(i+1)}\right\} \cap\{T>t\}$, as in Samaniego et al. [7] and the original coherent system signature in the set $\left\{T_{(n)} \leq t\right\}$ as in [2].

In our general setup, we consider the vector $T_{1}, \ldots, T_{n}$ of $n$ component lifetimes which are finite and positive random variables defined in a complete probability space $(\Omega, \mathfrak{I}, P)$, with $P\left(T_{i} \neq T_{j}\right)=1$, for all $i \neq j, i, j$ in $E=\{1, \cdots, n\}$, the index set of components. The lifetimes can be dependent but simultaneous failure are ruled out.

In what follows, to simplify the notation, we assume that relations such as $\subset,=, \leq,<, \neq$ between random variables and measurable sets, respectively, always hold with probability one, which means that the term $P$-a.s., is suppressed.

The evolution of components in time define a marked point process given through the failure times and the corresponding marks.

We denote by $T_{(1)}<T_{(2)}<\cdots<T_{(n)}$ the ordered lifetimes $T_{1}, T_{2}, \ldots, T_{n}$, as theyappear in time and by $X_{i}=\left\{j: T_{(i)}=T_{j}\right\}$ the corresponding marks. As a convention we set $T_{(n+1)}=T_{(n+2)}=\cdots=\infty$ and $X_{n+1}=X_{n+2}=\cdots=e$ where $e$ is a fictitious mark not in $E$. Therefore the sequence $\left(T_{(n)}, X_{n}\right)_{n \geq 1}$ defines a marked point process.

The mathematical formulation of our observations is given by a family of sub $\sigma$-algebras of $\mathfrak{I}$, denoted by

$$
\begin{aligned}
& \left(\mathfrak{I}_{t}\right)_{t \geq 0} \text {, where } \\
& \quad \mathfrak{I}_{t}=\sigma\left\{1_{\{T>s\}}, 1\left\{T_{(i)}>s\right\}\right. \\
& \left., X_{i}=j, 1 \leq j \leq n, j \in E, 0<s \leq t\right\}
\end{aligned}
$$

satisfies the Dellacherie ([8]),conditions of right continuity and completeness, and $T$ is the system lifetime

$$
T=\min _{1 \leq j \leq k} \max _{i \in K_{j}} T_{i}
$$

where $K_{j}, 1 \leq j \leq k$ are minimal cut sets, that is, a minimal set of components whose joint failure causes the system fail.

Intuitively, at each time $\mathrm{t}$ the observer knows if the events $\left\{T_{(i)} \leq t, X_{i}=j\right\}(\{T \leq t\})$ have either occurred or not and if they have, he knows exactly the value $T_{(i)}(T)$ and the mark $X_{i}$. We assumed that $T_{1}, \ldots, T_{n}$ are totally inaccessible $\mathfrak{I}_{t}$-stopping time. In a practical sense we can think of a totally inaccessible $\mathfrak{I}_{t}$ stopping time as an absolutely continuous lifetime.

The simple marked point process $N_{(i), j}(t)=1_{\left\{T_{(i)} \leq t, X=j_{i}\right\}}$ is an $\mathfrak{I}_{t}$-submartingale and from the Doob-Meyer decomposition we know that there exists a unique $\mathfrak{I}_{t}$ predictable process $\left(A_{(i), j}(t)\right)_{t \geq 0}$, called the $\mathfrak{I}_{t}$ compensator of $N_{(i), j}(t)$, with $A_{(i), j}(0)=0$ and such that $N_{(i), j}(t)-A_{(i), j}(t)$ is an $\mathfrak{J}_{t}$-martingale. $A_{(i), j}(t)$ is absolutely continuous by the totally inaccessibility of $T_{i}, 1 \leq i \leq n$. We also define the lifetime $T_{(i) j}$ through its process

$$
F_{(i) j}(t)=P\left(T_{(i) j} \leq t \mid \mathfrak{I}_{t}\right)=P\left(T_{(i)} \leq t, X_{i}=j \mid \mathfrak{I}_{t}\right)
$$

The compensator process is expressed in terms of the conditional probability, given the available information and generalize the classical notion of hazards. Intuitively, this corresponds to producing whether the failure is going to occur now, on the basis of all observations available up to, but not including, the present.

As $N_{(i), j}(t)$ can only count on the time interval $\left(T_{(i-1)}, T_{(i)}\right]$, the corresponding compensator differen 
tial $d A_{(i) j}(t)$ must vanish outside this interval. To count thei-th failure we let $N_{(i)}(t)=\sum_{j \geq 1} N_{(i) j}(t)$ with $\mathfrak{I}_{t}$ compensator process $A_{(i)}(t)=\sum_{j \geq 1} A_{(i) j}(t)$. The $\mathfrak{I}_{t}$ compensator of $N_{j}(t)=1_{\left\{T_{j} \leq t\right\}}$, corresponding to thejth components lifetime is $A_{j}(t)=\sum_{i \geq 1} A_{(i) j}(t)$.

Follows that the $\mathfrak{I}_{t}$-compensator of $N(t)=\sum_{i=1}^{n} \sum_{j=1}^{n} N_{(i) j}(t)$ is

$$
A(t)=\sum_{i=1}^{n} \sum_{j=1}^{n} A_{(i) j}(t) 1_{\left\{T_{(i-1)} \leq t<T_{(i)}\right\}}
$$

which is an $\mathfrak{I}_{t}$-predictable process and therefore unique ( see Bremaud ([9])).

Conveniently, we define the critical level of the component $j$ for the $i$-th failure, $Y_{(i) j}$, as the first time from which onwards the failure of component $j$ lead to system failure at $\left\{T=T_{(i)}, X_{i}=j\right\}$. We consider the $\mathfrak{I}_{t}$-compensator process $\left(A_{\varphi}(t)\right)_{t \geq 0}$ of the point process $N_{\varphi}(t)=1_{\{T \leq t\}}$, of the system lifetime $T$, such that $N_{\varphi}(t)-A_{\varphi}(t)$ is an zero mean $\mathfrak{I}_{t}$-martingale with $P(T \leq t)=E\left[N_{\varphi}(t)\right]=E\left[A_{\varphi}(t)\right]$. Bueno [6], proves the following results:

\section{Theorem 2.1.}

Under the above notation, in the set $\{T>t\}$, the $\mathfrak{I}_{t}$ compensator of $N_{\phi}(t)=1_{\{T \leq t\}}$, is

$$
A_{\varphi}(t)=\sum_{i=1}^{n} \sum_{j=1}^{n}[A(i) j(t)-A(i) j(Y(i) j)]^{+} 1_{\left\{T_{(i-1)} \leq t<T_{(i)}\right\}}
$$

where $a^{+}=\max \{a, 0\}$.

\section{Theorem 2.2.}

Let $T$ be the lifetime of a coherent system of ordern, with component lifetimes $T_{1}, \ldots, T_{n}$ which are totally inaccessible $T_{1}, \ldots, T_{n}$-stopping time. Then, under the above notation and at complete information level, we have

$$
\sum_{i=1}^{n} \sum_{j=1}^{n} \frac{P\left(T=T_{(i-1)}, X_{i}=j \mid \mathfrak{I}_{t}\right)}{P\left(T \geq T_{(i-1)} \mid \mathfrak{I}_{t}\right)} 1_{\left\{T_{(i-1)} \leq t<T_{(i)}\right\}}
$$

with $T_{(n+1)}=\infty$.

\section{Remarks 2.3.}

i) In the case of independent and identically distributed lifetimes we have

$$
P\left(T \leq t \mid \mathfrak{I}_{t}\right)=\sum_{i=1}^{n} \frac{P\left(T=T_{(i-1)}\right)}{P\left(T \geq T_{(i-1)}\right)} 1_{\left\{T_{(i-1)} \leq t<T_{(i)}\right\}}
$$

ii) Clearly, it is not seemingly true to think the general case of dependent components in the signatures context. However, as Navarro et al., [10], asked, it is plausible to analyse the case of dependent and identically distributed lifetimes ( any way, its holds true for exchangeable distribution). In this case we have

$$
P\left(T \leq t \mid \mathfrak{I}_{t}\right)=\sum_{i=1}^{n} \frac{P\left(T=T_{(i-1)} \mid \mathfrak{I}_{t}\right)}{P\left(T \geq T_{(i-1)} \mid \mathfrak{I}_{t}\right)} 1\left\{T_{(i-1)} \leq t<T_{(i)}\right\}
$$

Clearly, in the case of exchangeability, the expression in i) is holding.

\section{Corollary 2.4.}

Let $T$ be the lifetime of a coherent system of order $\mathrm{n}$, with component lifetimes $T_{1}, \ldots, T_{n}$ which are independent and identically distributed with continuous distribution $F$. Then,

$$
P\left(T \leq t \mid \mathfrak{I}_{t}\right)=\sum_{i=1}^{n} \beta_{i} 1\left\{T_{(i)} \leq t\right\}
$$

where

$$
\beta_{i}=\frac{P\left(T=T_{(i)}\right)}{P\left(T \geq T_{(i)}\right)}-\frac{P\left(T=T_{(i-1)}\right)}{P\left(T \geq T_{(i-1)}\right)}
$$

with $T_{(0)}=0, T_{(n+1)}=\infty, \beta_{i} \geq 0$ and $\sum_{i=1}^{n} \beta_{i}=1$.

\section{Definition 2.5.}

Let $T$ be the lifetime of a coherent system of order $n$, with component lifetimes $T_{1}, \ldots, T_{n}$ which are independent and identically distributed random variables with absolutely continuous distribution $F$. Then the dynamic signature vector $\beta$ is defined as

$$
\beta=\left(\beta_{1}, \cdots, \beta_{n}\right)
$$

where $\beta_{i}=\frac{P\left(T=T_{(i)}\right)}{P\left(T \geq T_{(i)}\right)}-\frac{P\left(T=T_{(i-1)}\right)}{P\left(T \geq T_{(i-1)}\right)}$ and the $T_{(i)}$ are the order statistics of $T_{i}, 1 \leq i \leq n$. 
Remarks 2.6.

We observe that

$$
\begin{aligned}
& \left.P\left(T=T_{(i)}\right)=\sum_{j=1}^{n} E\left[1\left\{T=T_{(i)}\right\}\right\}_{\left\{X_{i}=j\right\}}\right] \\
= & \sum_{j=1}^{n} E\left[1\left\{Y_{(i) j} \leq T_{(i) j}<T\right\}\right]=\sum_{j=1}^{n} P\left(Y_{(i) j} \leq T_{(i) j}<T\right) \\
= & \sum_{j=1}^{n}\left[F_{(i) j}(T)-F_{(i) j}\left(Y_{(i) j}\right)\right]
\end{aligned}
$$

\section{Minimal Repair and System Signature}

It is well known that there exists a bijective relation between the space of all distributions functions and the $\mathfrak{I}_{t}$-compensators space characterized by the so called Doléans exponential equation

$$
\bar{F}\left(t \mid \mathfrak{I}_{t}\right)=\mathrm{e}^{-A^{c}(t)} \pi_{s \leq t}(1+\Delta A(s))
$$

where $A^{c}(t)$ is the continuous part of $A(t)$ and $\Delta A(t)=A(t)-A^{c}(t)$ is its discrete part. Therefore, to detect the effects of the independent (exchangeable) lifetimes component minimal repairs in the ordered statistics and in the signatures $\beta_{i}$ itself, we are going to consider the minimal repair operation through compensator transform, as in Bueno [11].

\subsection{The First Minimal Repair Operation}

We are concerning with an improvement of the component lifetime Ti through a transformation of the $\mathfrak{I}_{t}-$ compensator process $A_{i}(t)$ of the counting process $N_{i}(t)=1_{\left\{T_{i} \leq t\right\}}$. The compensator process transform is in the form

$$
B_{i}(t)=\int_{0}^{t} \delta_{i}(s) \mathrm{d} A_{i}(s), 1 \leq i \leq n
$$

where $\left(\delta_{i}(s)\right)_{s} \geq 0$ is an $\mathfrak{I}_{t}$-predictable process. Clearly, if $0<\delta_{i}(s)<1$, the hazard process $\delta_{i}(s) \mathrm{d} A_{i}(s)$ is lower than the hazard process $\mathrm{d} A_{i}(s)$ and we understand such improvement of the components $i$ lifetime as a redundancy operation. The main tool in this approach is the Girsanov Theorem which proof is in [9](see A2. in Appendix).

In our particular case of minimal repair (see [11]) the compensator transform is in the form

$$
B_{i}(t)=\int_{0}^{t} \frac{A_{i}(s)}{1+A_{i}(s)} \mathrm{d} A_{i}(s)=A_{i}(t)-\ln \left(1+A_{i}(t)\right)
$$

in which case

$$
L_{\delta^{i}}(t)=\left[\frac{A_{i}\left(T_{i}\right)}{1+A_{i}\left(T_{i}\right)}\right]^{N_{i}(t)}\left(1+A_{i}(t)\right)
$$

It is remarkable (Norros,[12]) that the continuous components compensator processes at its final points, $A_{j}\left(T_{j}\right), 1 \leq j \leq n$, are independent and identically distributed random variables with standard exponential distribution. This holds no matter how dependent the actual lifetimes are and what the history, as long as si multaneous failures are ruled out. Therefore $E\left[A_{i}\left(T_{i}\right)\right]=1$ and we have

$$
L_{\delta^{i}}=\frac{\mathrm{d} Q_{\delta^{i}}}{\mathrm{~d} P}=A_{i}\left(T_{i}\right)
$$

It is well known (Arjas et al.[13]) that $A_{j}(t)=-\ln \bar{F}_{j}(t)$ where $F_{j}(t)=1-\bar{F}_{j}(t)=P\left(T_{j} \leq t \mid \mathfrak{\Im}_{t}\right)$ and the survival function of the component $\mathrm{j}$ after the compensator transform is

$$
\bar{F}_{j}(t)=\mathrm{e}^{-B_{j}(t)}=\mathrm{e}^{-A_{j}(t)+\ln \left(1+A_{j}(t)\right)}=\bar{F}_{j}(t)\left(1-\ln \bar{F}_{j}(t)\right)
$$

recovering the expression of the first Section.

At this point we can ask how the independent (exchangeable) lifetimes component minimal repairs affects the dependent ordered statistics and the signatures $\beta_{i}$ i itself. We remark that, in the following results, the proofs are heavily based in the fact that $\mathfrak{I}_{t}$ martingales summation is a $\mathfrak{I}_{t}$-martingale and the $\mathfrak{I}_{t}$ compensator is unique.

\section{Lemma 3.1.1.}

Let $A_{k}(t)$ be the $\mathfrak{I}_{t}$-compensator of $N_{k}(t)=1_{\left\{T_{k} \leq t\right\}}$ where $T_{k}$ is a totally inaccessible $\mathfrak{I}_{t}$-stopping time representing the lifetime of the component $k$. Under the minimal repair transform

$$
B_{k}(t)=\int_{0}^{t} \frac{A_{k}(s)}{1+A_{k}(s)} \mathrm{d} A_{k}(s)
$$

the $\mathfrak{J}_{t}$-compensator of thei-th failure, $A_{(i)}(t)$, un$\operatorname{der} Q_{\delta^{k}}$, is transformed to

$$
B_{(i)}(t)=\sum_{j=1}^{n} B_{(i) j}(t)
$$

where $B_{(i) j}(t)=A_{(i) j}(t)$ if $j \neq k$ and

$$
B_{(i), k}(t)=\int_{0}^{t} \frac{A_{(i) k}(s)}{1+A_{(i) k}(s)} \mathrm{d} A_{(i) k}(s)
$$


Proof

We observe that the $\mathfrak{I}_{t}$-compensator of the $i$-th failure is set as:

$$
\left.A_{(i)}(t)=\sum_{j=1}^{n} A_{(i) j}(t)=1_{\left\{T_{(i)} \leq t<T_{(i+1)}\right\}}\right\} \sum_{j=1}^{n} A_{(i) j}(t)
$$

Also, the component $\mathfrak{I}_{t}$-compensator can be set on the form:

$$
A_{j}(t)=\sum_{i=1}^{n} A_{(i) j}(t)=\sum_{j=1}^{n} 1\left\{T_{(i) \leq t<T}\right\} A_{(i+1)} A_{j}(t)
$$

In the case of a minimal repair transformation of the component $\mathrm{k}$, through its $\mathfrak{J}_{t}$-compensator we have:

$$
\begin{aligned}
B_{k}(t) & =\int_{0}^{t} \frac{A_{k}(s)}{1+A_{k}(s)} \mathrm{d} A_{k}(s)=\sum_{l=1}^{n} \int_{T_{(l-1)}}^{\left.T_{(l)}\right)^{t}} \frac{A_{k}(s)}{1+A_{k}(s)} \mathrm{d} A_{k}(s) \\
& =\sum_{l=1}^{n} \int_{T(l-1)}^{T_{(l)}^{\wedge t}} \frac{A_{(l) k}(s)}{1+A_{(l) k}(s)} \mathrm{d} A_{(l) k}(s)
\end{aligned}
$$

with $T_{(0)}=0$.

Therefore, the effect of the component $k$ minimal repair compensator transform, in the compensator of the $i$-th failure is through the $i$-th term of the last summation.

$$
\begin{aligned}
& B_{(i)}(t) \\
& =1\left\{T_{(i)} \leq t<T_{(i+1)}\right\}\left[\sum_{j=1, j \neq k}^{n} A_{(i) j}(t)+\int_{T_{(i-1)}}^{T_{(i)}^{\wedge t}} \frac{A_{(i) k}(s)}{1+A_{(i) k}(s)} \mathrm{d} A_{(i) k}(s)\right] \\
& =1\left\{T_{(i)} \leq t<T_{(i+1)}\right\} \\
& {\left[\sum_{j=1, j \neq k}^{n} A_{(i) j}(t)+\int_{T_{(i-1)}}^{T_{(i)}^{\wedge t}} \frac{A_{k}(s)}{1+A_{k}(s)} \mathrm{d} A_{k}(s)\right]}
\end{aligned}
$$

As $E\left[A_{k}\left(T_{k}\right)\right]=1$, by Girsanov Theorem, under the measure $\frac{\mathrm{d} Q_{\delta^{k}}}{\mathrm{~d} P}=A_{k}\left(T_{k}\right), B_{(i)}(t)$ is the $\mathfrak{I}_{t}$-compensator of $N_{(i)}(t)=1\left\{T_{(i)} \leq t\right\}$ and the effect of a minimal repair compensator transform, of the component $k$, in the compensator of the $i$-th failure is

$$
B_{(i) k}(t)=\int \frac{A_{(i) k}(s)}{1+A_{(i) k}(s)} \mathrm{d} A_{(i) k}(s)
$$

In view of the identically (exchangeability) distribution component lifetimes conditions in the signatures definition we must consider the minimal repair operations in all component lifetimes under the measure $Q_{\delta}$ defined by the Radon Nikodym derivative

$$
\mathrm{d} Q_{\delta} / \mathrm{d} P=\pi_{k=1}^{n} A_{k}\left(T_{k}\right)
$$

and we have, using Girsanov Theorem, the following result:

\section{Corollary 3.1.2.}

Let $A_{j}(t)$ be the $\mathfrak{I}_{t}$-compensator of $N_{j}(t)=1\left\{T_{j} \leq t\right\}$ where $T_{j}$ is a totally inaccessible $\mathfrak{J}_{t}$-stopping time representing the lifetime of the componentj. Under the minimal repair transform

$$
B_{j}(t)=\int_{0}^{t} \frac{A_{j}(s)}{1+A_{j}(s)} \mathrm{d} A_{j}(s), 1 \leq j \leq n
$$

the $\mathfrak{I}_{t}$-compensator of the $i$-th failure, $A_{(i)}(t)$, under $Q_{\delta}$, is transformed in

$$
B_{(i)}(t)=\sum_{k=1}^{n} B_{(i) k}(t)
$$

where

$$
B_{(i) k}(t)=\int \frac{A_{(i) k}(s)}{1+A_{(i) k}(s)} \mathrm{d} A_{(i) k}(s)
$$

\section{Theorem 3.1.3.}

Let $T_{1}, T_{2}, \ldots, T_{n}$ be totally inaccessible $\mathfrak{J}_{t}$-stopping time representing the lifetimes of ancomponent coherent system with lifetime $T$, which are absolutely continuous independent and identically distributed. Then, under the minimal repair transformation of all component lifetimes we have:

$$
Q_{\delta}(T \leq t)=\sum_{i=1}^{n} \beta_{i}^{*} Q_{\delta}\left(T_{(i)} \leq t\right)
$$

where

$$
\begin{gathered}
\beta_{i}^{*}=\frac{Q_{\delta}\left(T=T_{(i)}\right)}{Q_{\delta}\left(T \geq T_{(i)}\right)}-\frac{Q_{\delta}\left(T=T_{(i-1)}\right)}{Q_{\delta}\left(T \geq T_{(i-1)}\right)} ; \\
Q_{\delta}\left(T=T_{(i)}\right)=\sum_{k=1}^{n} \bar{F}_{(i) k}\left(Y_{(i) k}\right)\left(1-\ln \bar{F}_{(i) k}\left(Y_{(i) k}\right)\right) \\
-\bar{F}_{(i) k}\left(T_{(i) k}\right)\left(1-\ln \bar{F}_{(i) k}\left(T_{(i) k}\right)\right) \\
Q_{\delta}\left(T_{(i)} \leq t\right)=\sum_{k=1}^{n} 1-\bar{F}_{(i) k}(t)\left(1-\bar{F}_{(i) k}(t)\right)
\end{gathered}
$$

and $\mathrm{d} Q_{\delta} / \mathrm{d} P=\pi_{k=1}^{n} A_{k}\left(T_{k}\right)$

Proof

Firstly, we note that, under $Q_{\delta}$, the lifetimes are independent:

$$
\begin{aligned}
& Q_{\delta}\left(T_{1} \leq t_{1}, \ldots, T_{n} \leq t_{n}\right) \\
= & E\left[\pi_{k=1}^{n} A_{k}\left(T_{k}\right) 1_{\left\{T_{k} \leq t_{k}\right\}}\right]=\pi_{k=1}^{n} E\left[A_{k}\left(T_{k}\right) 1_{\left\{T_{k} \leq t_{k}\right\}}\right] \\
= & \underset{k}{\pi} E_{\delta^{k}}\left[1_{\left\{T_{k} \leq t_{k}\right\}}\right]=\pi_{k=1}^{n} Q_{\delta^{k}}\left(T_{k} \leq t_{k}\right)=\pi_{k=1}^{n} Q_{\delta}\left(T_{k} \leq t_{k}\right)
\end{aligned}
$$


Also, as $T_{j}, 1 \leq j \leq n$ are identically distributed, the $\mathfrak{I}_{t}$-compensators $A_{j}(t)$, of $1_{\left\{T_{j} \leq t\right\}}$ are identical and we conclude that the $T_{i}$ lifetimes are identically distributed under $Q_{\delta}$. Therefore the signature decomposition under $Q_{\delta}$ remains true.

Furthermore

$$
\begin{aligned}
Q_{\delta}\left(T=T_{i}\right) & =E_{Q_{\delta}}\left(1_{\left\{T=T_{i}\right\}}\right) \\
& =E\left[\prod_{k=1}^{n} A_{k}\left(T_{k}\right) 1_{\left\{T=T_{i}\right\}}\right] \\
& =\sum_{j=1} E\left[\prod_{k=1}^{n} A_{k}\left(T_{k}\right) 1_{\left\{T=T_{i}\right\}} 1_{\left\{X_{i}=j\right\}}\right] \\
& =\sum_{j=1}^{n} E\left[\prod_{k=1}^{n} A_{k}\left(T_{k}\right) 1_{\left\{T=T(i) j_{i}\right\}}\right] \\
& =\sum_{j=1}^{n} E\left[\prod_{k=1}^{n} A_{k}\left(T_{k}\right) 1_{\left\{A_{j}(t)=A_{j}\left(T_{(i) j}\right)\right\}}\right] \\
& =\sum_{j=1}^{n} E\left[\prod_{k=1, k \neq j}^{n} A_{k}\left(T_{k}\right)\right] E\left[A_{j}\left(T_{j}\right) 1\left\{A_{j}(t)=A_{j}\left(T_{(i) j}\right)\right\}\right] \\
& =\sum_{j=1}^{n} E\left[A_{j}\left(T_{j}\right) 1_{\left.\left\{A_{j}(t)=A_{j}\left(T_{(i) j}\right)\right\}\right]}\right]
\end{aligned}
$$

The equivalence in the third equality is justified in Norros [12] which defines the $P$ a:s: inverse of $A_{j}(t)$.As, under the hypothesis, $A_{j}(t)=-\ln \bar{F}_{(i) j}(t)$ where $F_{(i) j}(t)=p\left(T_{(i) j} \leq t \mid \mathfrak{I}_{t}\right)$ we have:

$$
\begin{aligned}
& E\left[A_{j}\left(T_{j}\right) 1\left\{T_{\left.=T_{(i) j}\right\}}\right]\right. \\
= & E\left[A_{j}\left(T_{j}\right) 1\left\{_{\left\{Y_{(i) j} \leq T_{(i) j}<T\right\}}\right]=\int_{-\ln \bar{F}_{(i) j}\left(Y_{(i) j}\right)}^{-\ln \bar{F}_{(i)}(T)} x e^{-x} \mathrm{~d} x\right. \\
= & \bar{F}_{(i) j}\left(Y_{(i) j}\right)\left(1-\ln \bar{F}_{(i) j}\left(Y_{(i) j}\right)\right)-\bar{F}_{(i) j}(T)\left(1-\ln \bar{F}_{(i) j}(T)\right)
\end{aligned}
$$

Therefore

$$
\begin{aligned}
& Q_{\delta}\left(T=T_{i}\right)= \\
& \sum_{j=1}^{n} \bar{F}_{(i) j}\left(Y_{(i) j}\right)\left(1-\ln \bar{F}_{(i) j}\left(Y_{(i) j}\right)\right)-\bar{F}_{(i) j}(T)\left(1-\ln \bar{F}_{(i) j}(T)\right)
\end{aligned}
$$

Also, we have

$$
\begin{aligned}
& Q_{\delta}\left(T_{(i)} \leq t\right)=E_{Q_{\delta}}\left(1_{\left\{T_{(i)_{i}} \leq t\right\}}\right)=\sum_{j=1}^{n} E\left[\prod_{k=1}^{n} A_{k}\left(T_{k}\right) 1_{\left\{T_{(i)} \leq t\right\}}\right] \\
& =\sum_{j=1}^{n} E\left[\prod_{k=1, k \neq j}^{n} A_{k}\left(T_{k}\right)\right] E\left[A_{j}\left(T_{j}\right) 1_{\left\{A_{j}\left(T_{(i) j}\right) \leq A_{j}(t)\right\}}\right] \\
& =\sum_{j=1}^{n} E\left[A_{j}\left(T_{j}\right) 1_{\left\{A_{j}\left(T_{(i) j}\right) \leq A_{j}(t)\right\}}\right]
\end{aligned}
$$

$$
\begin{aligned}
& E\left[A_{j}\left(T_{j}\right) 1\left\{A_{j}\left(T_{(i) j}\right) \leq A_{j}(t)\right\}\right] \\
= & \int_{0}^{-\ln \bar{F}_{(i) j}(t)} x \mathrm{e}^{-x} \mathrm{~d} x=1-\bar{F}_{(i) j}(t)\left(1-\ln \bar{F}_{(i) j}(t)\right)
\end{aligned}
$$

and

$$
Q_{\delta}\left(T_{(i)_{i}} \leq t\right)=\sum_{j=1}^{n} 1-\bar{F}_{(i) j}(t)\left(1-\overline{\ln F}_{(i) j}(t)\right)
$$

\subsection{Successive Minimal Repairs}

More generally, we intend to define a measure $Q_{\delta^{i}}^{n}$ which is obtained when $T_{i}$ is deferred $\mathrm{n}$ times in the sense of minimal repair. The measures $Q_{\delta^{i}}^{0}$, $Q_{\delta^{i}}^{1}, \cdots, Q_{\delta^{i}}^{n}$ are defined successively by $Q_{\delta^{i}}^{0}=P$ and

$$
Q_{\delta^{i}}^{n+1}(.)=\int_{\Omega} P\left(. \mid \mathfrak{I}_{T_{i-}}\right)(w) \mathrm{d} Q_{\delta^{i}}^{n}(w)
$$

It can be proved that, for any $\mathrm{n}$, the probability measure $Q_{\delta^{i}}^{n} i$ is absolutely continuous with respect to $P$, with Radon Nikodyn derivative

$$
\mathrm{d} Q_{\delta^{i}}^{n} / \mathrm{d} P=\frac{1}{n !} A_{i}\left(T_{i}\right)^{n}
$$

We reason as follows:

Suppose that we choose an w with probability distribution $P$ and starting proceeding at time 0 . Suddenly $T_{i}$ occurs. In order to make a minimal repair, we have to change our $w$ to another, say $w_{0}$ which is indistinguishable from $w$ strictly before the time $T_{i}(w)$ and satisfies $T_{i}\left(w_{0}\right)>T_{i}(w)$. Moreover, $w_{0}$ should be chosen according to an appropriated distribution among the candidates satisfying these conditions. Indeed we choose $w_{0}$ accordingto $P\left(. \mid \mathfrak{J}_{T_{i}^{-}}\right)$, the value of the process $P\left(. \mid \mathfrak{I}_{t^{-}}\right)$at $T_{i}$, where

$$
\mathfrak{I}_{T_{i}^{-}}=\sigma\left\{A \cap\left\{t<T_{i}\right\}, A \in \mathfrak{I}_{t}, t \geq 0\right\}
$$

is the history strictly before $T_{i}$ Thus choosing $w_{0}$ according to $P\left(. \mid \mathfrak{I}_{T_{i}^{-}}\right)$we may proceed further as if nothing had happened.

Intuitively, if $S$ is an $\mathfrak{I}_{t}$-stopping time, the difference between the $\sigma$-algebras $\mathfrak{I}_{S_{-}}$and $\mathfrak{I}_{S}$ is that, in $\mathfrak{I}_{S_{-}}$it is

known when $S$ occurs, but its not known what else happen at time $S$. For example, if $\mathrm{S}$ is the failure time of a systems component, it is known in $\mathfrak{I}_{S_{-}}$, but at this time, we do not known what component will fail.

Indeed, as in Section 3.1, its holds for $n=1$. Suppose that its holds for somen fixed.We have to prove that:

$$
E_{Q_{\delta^{i}}^{n+1}}[S]=E\left[S \frac{1}{n+1 !} A_{i}\left(T_{i}\right)^{n+1}\right]
$$

for any random variable $S, \mathfrak{J}_{T_{i-}}$-measurable.

And 


$$
\begin{aligned}
E_{Q_{\delta^{i}}^{n+1}}[S] & =E_{Q_{\delta^{i}}^{n+1}}\left[E\left(S \mid \mathfrak{I}_{T_{i-}}\right)\right]=E\left[\frac{1}{n !} A_{i}\left(T_{i}\right)^{n} E\left(S \mid \mathfrak{I}_{T_{i-}}\right)\right] \\
& =E\left[\int_{0}^{\infty} \frac{1}{n !} A_{i}(t)^{n} E\left(S \mid \mathfrak{J}_{t_{i-}}\right) \mathrm{d} 1_{\left\{T \leq t_{i}\right\}}\right] \\
& =E\left[\int_{0}^{\infty} \frac{1}{n !} A_{i}(t)^{n} E\left(S \mid \mathfrak{\Im}_{t_{i-}}\right) \mathrm{d} A_{i}(t)\right] \\
& =E\left[\int_{0}^{\infty} \frac{1}{(n+1) !} E\left(S \mid \mathfrak{I}_{t_{i-}}\right) \mathrm{d} A_{i}(t)^{n+1}\right] \\
& =E\left[E\left(S \mid \mathfrak{\Im}_{T_{i}}\right) \frac{1}{(n+1) !} A_{i}\left(T_{i}\right)^{n+1}\right]
\end{aligned}
$$

As the process $\frac{1}{n !} A_{i}(t)^{n} E\left(S \mid \mathfrak{\Im}_{t_{i-}}\right)$ is $\mathfrak{\Im}_{t}$-predictable, the forth above equality is true.The sixth equality follows from Dellacheries integration formula.

$$
E\left[E\left(S \frac{1}{(n+1) !} A_{i}\left(T_{i}\right)^{n+1} \mid \mathfrak{J}_{T_{i}}\right)\right]=E\left[S \frac{1}{(n+1) !} A_{i}\left(T_{i}\right)^{n+1}\right]
$$

Furthermore we have

$$
\begin{aligned}
& Q_{\delta^{i=}}^{n}\left(T_{i}>t\right)=E Q_{\delta^{\delta_{i}}}^{n}\left[1_{\left\{T_{i}>t\right\}}\right]=E\left[\frac{1}{n !} A_{i}\left(T_{i}\right)^{n}{ }_{\left\{T_{i}>t\right\}}\right] \\
& =E\left[\frac{1}{n !} A_{i}\left(T_{i}\right)^{n} 1_{\left\{A_{i}\left(T_{i}\right)>A_{i}(t)\right\}}\right]=\int_{A_{i}(t)}^{\infty} \frac{x^{n}}{n !} \mathrm{e}^{-x} \mathrm{~d} x=\sum_{l=0}^{n-1} \frac{A_{i}(t)^{l} \mathrm{e}^{-A_{i}(t)}}{i !}
\end{aligned}
$$

and we conclude that the number of minimal repairs occurring before $T_{i}$ is modeled by a doubly stochastic Poisson process.

Next we consider the realization of an equal and finite number of minimal repairs of each totally inaccessible $\mathfrak{I}_{t}$-stopping time representing the components lifetimes. Asthe continuous components compensator processes at its final points, $A_{i}\left(T_{i}\right), 1 \leq i \leq n$, are independent and identically distributed random variables with standard exponential distribution, in the case of a fixed configuration $m=(m, \ldots, m)$, where $m$ is the number of minimal repairs of componenti, we can define a product probability measure in $\Omega \times \Omega \times \ldots \times \Omega$

$$
Q^{m}=\pi_{i=1}^{m} Q_{\delta^{i}}^{m}
$$

where $\mathrm{d} Q_{\delta^{i}}^{m} / \mathrm{d} P=\frac{1}{m} A_{i}\left(T_{i}\right)^{m}$.

Follows that, under the corresponding $\mathfrak{I}_{t}$-compensator transform we can enunciate the Theorem.

\section{Theorem 3.2.3.}

Let $T_{1}, \ldots, T_{n}$ be totally inaccessible $\mathfrak{I}_{t}$-stopping time representing the lifetimes of an component coherent system with lifetime $T$. Then, under the minimal repair configuration $\mathrm{m}$ and under the probability measure $Q^{m}$, we have

$$
Q^{m}(T \leq t)=\sum_{i=1}^{n} Q_{i}^{m}\left(T_{i} \leq t\right)
$$

Where

$$
\beta_{i}^{*}=\frac{Q^{m}\left(T=T_{(i)}\right)}{Q^{m}\left(T \geq T_{(i)}\right)}-\frac{Q^{m}\left(T=T_{(i-1)}\right)}{Q^{m}\left(T \geq T_{(i-1)}\right)}
$$

\section{Conclusions}

In this paper we get resultsin a general set up in which a coherent system can be set as a stochastic process including the order statistics in the signature context. The main difference between this results and the previous one in the signature field is the dynamic aspect. In this setting we can realize redundancy oprations and ask about the reliability component importance. The main question is to clear out how a component operation affect the order statistics in the signature representation. The compensator transforms can help to answer such a question. Note that, even if the components are independent and identically distributed, the order statistics are not. We conclude that, in the general setting, we can develop some classical properties in reliability theory.

\section{Acknowledgements}

This work was partially supported by FAPESP, Proc. No. 2010/52227-0

\section{References}

[1] Arjas, E. and Yashin, A. (1988). A note on randonm intensities and conditional survivalfunctions. Journal of Applied probability. 25, 630 - 635.doi:10.2307/3213991

[2] Aven, T. and Jensen, U. (1999). Stochastic Models ineliability. Springer Verlag, New York. doi:10.1007/b97596

[3] Barlow and Proschan,F. (1981). Statistical Theory of Reliability and Life Testing: Probability models. Hold, Reinhart and Wiston, Inc. Silver Spring, MD.

[4] Bremaud ,P. (1981). Point Processes and Queues: Martingale Dynamics.Springer-Verlag, New York.

[5] Bueno, V.C. (2005). Minimal standby redundancy allocation in a K-out-of-n:F systemof dependent components. European Journal of Operation Research. 165, 786-793.doi:10.1016/j.ejor.2003.01.004

[6] Bueno, V.C. (2010). Dynamics signature of a coherent system. Submited.S ao PauloUniversity, S ao Paulo, Brazil. 
[7] Kochar, S., Mukherjee, H., Samaniego, F.(1999). The signature of a coherent systemand its application to comparisons among systems. Naval Research Logistic. 46, $507-523$.

doi:10.1002/(SICI)1520-6750(199908)46:5<507::AIDNAV4>3.0.CO;2-D

[8] Navarro, J., Balakrishnan, N. and Samaniego, F.J. (2008). Mixture representation ofresidual lifetimes of used systems. Journal of Applied Probability. 45, 1097 -1112.doi:10.1239/jap/1231340236

[9] Norros, I. (1986). A compensator representation of multivariate life length distributions, with applications. Scand. J. Statist. 13, 99-112.

[10] Samaniego, F. (1985). On closure of the IFR class un-

\section{Appendix}

\section{A1. Stopping time}

An extended and positive random variable $\tau$ is an $\mathfrak{I}_{t}$ stopping time if, and only if, $\{\tau \leq t\}$ for all $t \geq 0$; an $\mathfrak{I}_{t}$-stopping time $\tau$ is called predictable if an increasing sequence $\left(\tau_{n}\right)_{n \geq 0}$ of $\mathfrak{I}_{t}$-stopping time, $\tau_{n}<\tau$, exists such that $\tau_{n} \rightarrow \tau$ as $n \rightarrow \infty$; an $\mathfrak{I}_{t}$-stopping time $\tau$ is totally inaccessible if $P(\tau=\sigma<\infty)=0$ for all predictable $\mathfrak{I}_{t}$-stopping time $\sigma$. For a mathematical basis of stochastic processes applied to reliability theory seethe book of Aven and Jensen [14].

\section{A2. Theorem A.2 (Girsanov)}

Let $T_{i}, \quad 1 \leq i \leq n$ be totally inaccessible $\mathfrak{I}_{t}$ stoppingtimes, the point processes $N_{i}(t)=1_{\left\{T_{i} \leq t\right\}}$, der formation of coherent systems. IEEE Transactions in Reliability. R-34, 69-72.doi:10.1109/TR.1985.5221935

[11] Samaniego,F.J. (2007). System signatures and their applications in engineering re-liability. International Series in Operation Research and Management Science, Vol 110, Springer, New York.

[12] Samaniego, F.J., Navarro, J. and Balakrishnan, N. (2009). Dynamic signatures andtheir in comparing the reliability of a new and used systems. Naval Research Logistic. 56, 577-596.doi:10.1002/nav.20370

[13] Shaked, M., Suarez-Llorens, A. (2003). On the comparison of reliability experimentsbased on the convolution order. Journal of American Statistical Association. 98, 693-702.doi:10.1198/016214503000000602

with $\mathfrak{I}_{t}$-compensators $A_{i}(t), 1 \leq i \leq n$. Let $\left(\delta_{i}(t)\right)_{t \geq 0}$, be non negative $\mathfrak{I}_{t}$-predictable processes such that for all $t \geq 0$ and for all $\quad 1 \leq i \leq n \quad, \quad B_{i}(t)=\int_{0}^{t} \delta_{i}(s) \mathrm{d} A_{i}(s)<\infty, \quad$ With $\delta(t)=\left(\delta_{1}(t), \ldots, \delta_{n}(t)\right)$ and $\delta(\infty)=\delta$. Then

$$
L_{\delta(t)}=\prod_{i=1}^{n}\left[\delta_{i}\left(T_{i}\right)\right]^{N_{i}(t)} \mathrm{e}^{A_{i}(t)-B_{i}(t)}
$$

is a non negative $\mathfrak{I}_{t}$-local martingale and a non negative $\mathfrak{I}_{t}$-super martingale.

Furthermore, if $E\left[L_{\delta}\right]=1$, the probability measure $Q_{\delta}$ defined by the Radon Nikodym derivative $\mathrm{d} Q_{\delta} / \mathrm{d} P=L_{\delta}$ is such that, under $Q_{\delta}, B_{i}(t)$ is the unique $\mathfrak{I}_{t}$-compen- sator process of $N_{i}(t)$.

In particular, we denote $\delta^{i}=\left(1, \cdots, 1, \delta_{\infty}(i), 1, \cdots, 1\right)$ and $\mathrm{d} Q_{\delta^{i}} / \mathrm{d} P=L_{\delta^{i}}$. 